ARTICLE

\title{
Ferroelectric phase-transition frustration near a tricritical composition point
}

\author{
Xian-Kui Wei (1) 1,6凶, Sergei Prokhorenko (1) 2,6, Bi-Xia Wang (1) 3, Zenghui Liu (1) ${ }^{4}$, Yu-Juan Xie ${ }^{3}$, \\ Yousra Nahas (iD) ${ }^{2}$, Chun-Lin Jia ${ }^{1,5}$, Rafal E. Dunin-Borkowski ${ }^{1}$, Joachim Mayer ${ }^{1}$, Laurent Bellaiche (iD) ${ }^{2 \otimes} \&$ \\ Zuo-Guang Ye iD ${ }^{3 凶}$
}

Phase transition describes a mutational behavior of matter states at a critical transition temperature or external field. Despite the phase-transition orders are well sorted by classic thermodynamic theory, ambiguous situations interposed between the first- and second-order transitions were exposed one after another. Here, we report discovery of phase-transition frustration near a tricritical composition point in ferroelectric $\mathrm{Pb}\left(\mathrm{Zr}_{1-\mathrm{x}} \mathrm{Ti}_{\mathrm{x}}\right) \mathrm{O}_{3}$. Our multi-scale transmission electron microscopy characterization reveals a number of geometrically frustrated microstructure features such as self-assembled hierarchical domain structure, degeneracy of mesoscale domain tetragonality and decoupled polarization-strain relationship. Associated with deviation from the classic mean-field theory, dielectric critical exponent anomalies and temperature dependent birefringence data unveil that the frustrated transition order stems from intricate competition of short-range polar orders and their decoupling to long-range lattice deformation. With supports from effective Hamiltonian Monte Carlo simulations, our findings point out a potentially universal mechanism to comprehend the abnormal critical phenomena occurring in phase-transition materials.

\footnotetext{
${ }^{1}$ Ernst Ruska-Centre for Microscopy and Spectroscopy with Electrons, Forschungszentrum Jülich GmbH, Jülich, Germany. ${ }^{2}$ Physics Department and Institute for Nanoscience and Engineering, University of Arkansas, Fayetteville, NC, USA. ${ }^{3}$ Department of Chemistry and 4D LABS, Simon Fraser University, Burnaby, BC, Canada. ${ }^{4}$ Electronic Materials Research Laboratory, Key Laboratory of the Ministry of Education and International Center for Dielectric Research, Xi'an Jiaotong University, Xi'an, China. ${ }^{5}$ School of Microelectronics, Xi'an Jiaotong University, Xi'an, China. ${ }^{6}$ These authors contributed equally: Xian-Kui Wei,

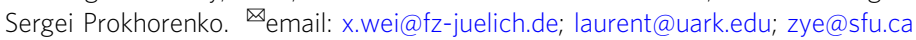


A s a time-honored research topic, phase transition (PhT) covers a broad range of intriguing physical phenomena such as giant electromechanical response ${ }^{1,2}$, magnetoelectric multiferroicity ${ }^{3}$, superconductivity ${ }^{4}$, etc. The classic thermodynamic theory 5 expounds that the first- and second-order transitions are characteristic of specific signatures such as latent heat, volume change and divergent correlation length. However, an affirmative judgment on order of the transition cannot be made for cases with ambiguous situations ${ }^{6-9}$, which therefore leaves an unfilled gap with the existing theory ${ }^{10-12}$. Geometric frustration depicts an intrinsic incompatibility of some fundamental interactions with respect to the underlying lattice geometry. Stimulated by this, a plethora of unusual phenomena and intriguing effects have been reported, e.g., spin liquids and spin ice ${ }^{13}$, glass-tocrystalline transitions $^{14}$, and exotic spiral ferroelectric states ${ }^{15,16}$. Given their commonality in phenomenological abnormality about $\mathrm{PhT}$, a potential competition between the first- and second-order transitions at the tricritical point of ferroelectric $\mathrm{Pb}\left(\mathrm{Zr}_{1-x} \mathrm{Ti}_{x}\right) \mathrm{O}_{3}$ (PZT, $x \geq 0.50$ ) is investigated in this work.

\section{Results}

Mesoscopic-scale structural anomaly. Experimental and theoretical studies have revealed that there exist three tricritical points in the phase diagram of PZT (Fig. 1a). Given the complex structural evolution on the rhombohedral side $(0.06 \leq$ $\left.x_{\mathrm{tcr}}^{\mathrm{R}} \leq 0.26\right)^{17,18}$ and at the morphotropic phase boundary (MPB, $\left.x_{\mathrm{tcr}}^{\mathrm{tr}} \approx 0.45\right)^{12,19,20}$, we hereby focus our attention on the tetragonal-side tricritical point, $x_{\mathrm{tcr}}^{\mathrm{T}}$, which was reported to locate in the $0.6 \leq x_{\mathrm{tcr}}^{\mathrm{T}} \leq 0.7$ composition range $\mathrm{r}^{12,17,21}$. By probing temperature-dependent spontaneous polarization $\left(P_{\mathrm{S}}\right)$, dielectric constant $(\varepsilon)$, and lattice tetragonality ( $c / a$ ratio), a continuous-todiscontinuous transition of the physical quantities at Curie temperature $\left(T_{\mathrm{C}}\right)$ evidences that the second-order transition changes to the first-order one at $x_{\mathrm{tcr}}^{\mathrm{T}} \approx 0.65$ with increasing $x$ (Fig. $1 \mathrm{~b}$ and Supplementary Fig. 1). On this basis, the correlation of ferroelectric domain morphology with the $\mathrm{PhT}$ order is probed using dark-field transmission electron microscopy (TEM). To distinguish the domain polarization orientation, the failure of Friedel's law due to dynamical scattering 22 is considered, i.e., the domains show bright contrast once the $\boldsymbol{P} . \boldsymbol{g}>0\left(\boldsymbol{P}\right.$, a component of $\boldsymbol{P}_{\boldsymbol{S}} ; \boldsymbol{g}$, scattering vector) criterion is satisfied under two-beam conditions.

We find that the PZT crystals with $x<x_{\mathrm{tcr}}^{\mathrm{T}}$ are characteristic of nesting ferroelectric tetragonal $\left(\mathrm{FE}_{\mathrm{T}}\right)$ and monoclinic $\left(\mathrm{FE}_{\mathrm{M}}\right)$ domains at nanometer scale (Fig. 1c and Supplementary Fig. 2). In sharp contrast, the $x=0.65$ crystal is comprised of regularly arranged wide $c$ domains $(\sim 1 \mu \mathrm{m})$ and narrow $a$ domains $(\sim 100 \mathrm{~nm})$, which is analogous to $\mathrm{PbTiO}_{3}$ (refs. $\left.{ }^{23,24}\right)$ undergoing the first-order transition (Fig. 1d). However, diffraction contrast analysis unveils that the ferroelastic domains are composed of self-assembled $\mathrm{FE}_{\mathrm{T}}$ and $\mathrm{FE}_{\mathrm{M}}$ nanodomains (inset in Fig. 1d). Being consistent with the neutron diffraction result ${ }^{25}$ at $x<x_{\text {tcr }}^{\mathrm{T}}$, our effective Hamiltonian Monte Carlo simulations further reveal that the structural phase of first-order-transition PZT is dominated by the $\mathrm{FE}_{\mathrm{T}}$ phase at $x>x_{\mathrm{tcr}}^{\mathrm{T}}$ (Supplementary Fig. 3). Given the stable coexistence of different phases at temperature
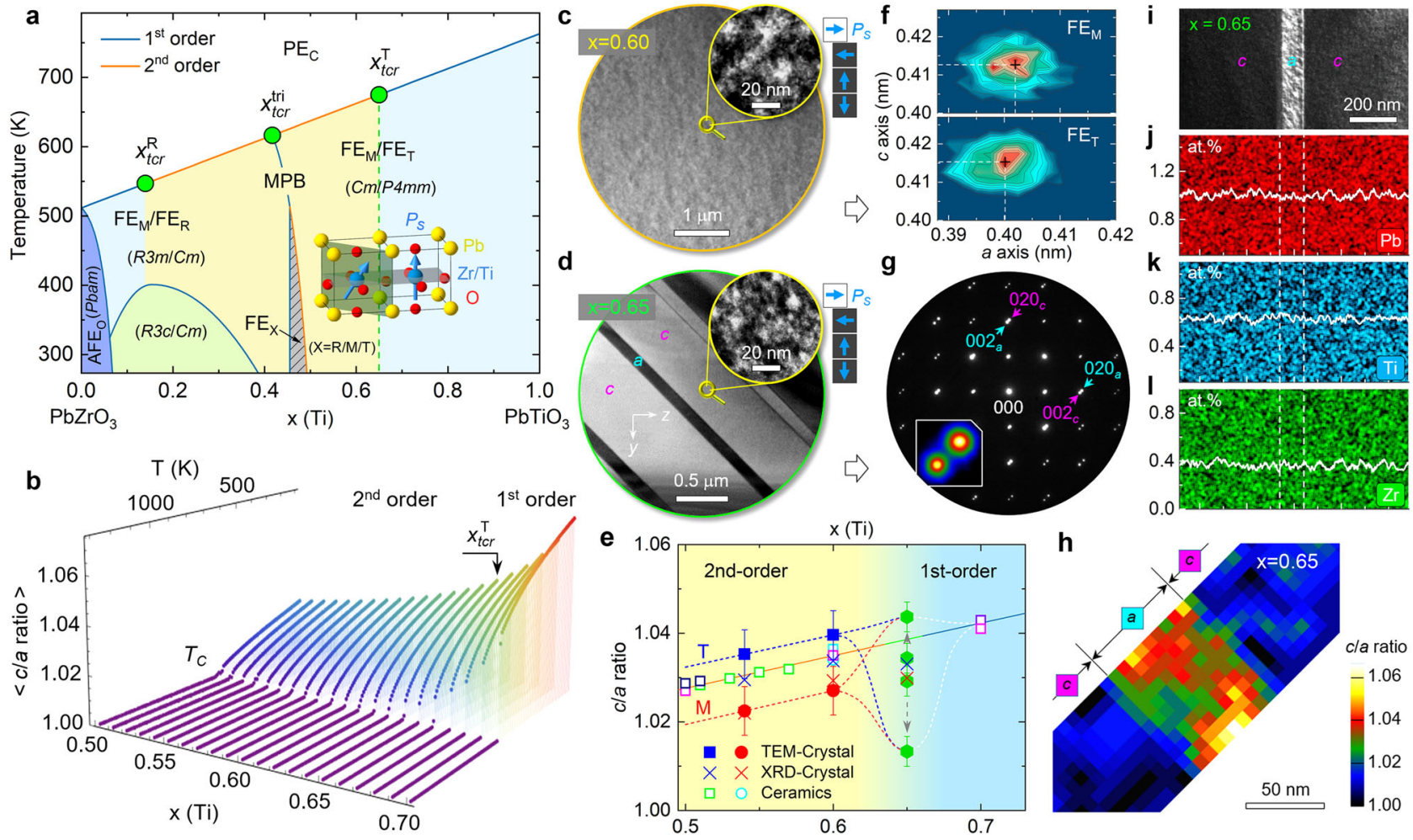

Fig. 1 Phase diagram, domain structure, and lattice tetragonality. a Schematic phase diagram of $P Z T\left(P E_{C}-\right.$ paraelectric, $A F E_{O}-$ antiferroelectric, $F E_{R}-$ rhombohedral) with annotation of tricritical points $\left(x_{t c r}^{R}, x_{t c r}^{t r i}, x_{t c r}^{T}\right.$ : solid green circle) and $\mathrm{FE}_{\mathrm{M}} / \mathrm{FE}_{\mathrm{T}}$ unit cell. $\mathbf{b}$ Temperature dependent average $c / a$ ratio obtained by effective Hamiltonian Monte Carlo simulations with $x_{\text {tcr }}^{\top} \approx 0.65$. $\mathbf{c}$, d Bright- and dark-field $\left(\boldsymbol{g}=020_{T}\right)$ TEM images (inset) of $x=0.60$ and 0.65 crystals recorded along $[100]_{T}$ zone axis, respectively. e Phase tetragonality-composition diagram and postulated traces of $\mathrm{FE}_{\mathrm{T}}$ (blue) and $\mathrm{FE}_{\mathrm{M}}$ (red) phases at room temperature. The gray arrows denote changes of ferroelastic domain tetragonality with time at $x_{\text {tcr }}^{\top}$ and the error bars are s.d. $\mathbf{f} 2 \mathrm{D}$ lattice parameter plot for $x=0.60$ crystal extracted from high-resolution TEM image with $(c / a)_{T}=1.038$ and $(c / a)_{M}=1.027$. g-I SAED pattern along $[100]_{T}$ direction, representative $(020)_{a} /(002)_{c}$ reflections (inset, log plot), 2D c/a ratio map, dark-field image, elemental maps, and line profiles of $x=0.65$ crystal, respectively. The $\mathrm{Zr} / \mathrm{Ti}$ molar ratio is measured as $\mathrm{Zr}: \mathrm{Ti}=37: 63$. 
$t<T_{\mathrm{C}}$, the unique hierarchical domain configuration observed in $x_{\mathrm{tcr}}^{\mathrm{T}}=0.65$ crystal therefore implies a geometric frustration between the PhT orders.

For the $x<x_{\mathrm{tcr}}^{\mathrm{T}}$ crystals, analysis on $\mathrm{X}$-ray diffraction data shows that lattice tetragonality of the $\mathrm{FE}_{\mathrm{T}}$ phase is larger than that of the $\mathrm{FE}_{\mathrm{M}}$ phase, which agrees well with the data of PZT ceramics $^{25-28}$ and is corroborated by our TEM data obtained from local regions (Fig. 1e, f). For the $x=0.65$ crystal, the $c / a$ ratio of the tetragonal phase is also suggested to be larger than the monoclinic phase, $(c / a)_{\mathrm{T}}=1.033$ and $(c / a)_{\mathrm{M}}=1.030$, both of which are smaller than the expected values in the overall trend. However, our selected area electron diffraction (SAED) experiments surprisingly reveal that the two distinct lattice ratios stem from the ferroelastic $a$ and $c$ domains separately, which are measured as $(c / a)_{a}=1.034$ and $(c / a)_{c}=1.029$. After month-level storage, the domain lattice ratio is further increased to $(c / a)_{a}=$ $1.044 \pm 0.003$ and decreased to $(c / a)_{c}=1.014 \pm 0.003$, which is confirmed by real-space mapping using the $4 \mathrm{D}$ scanning TEM technique (Fig. 1g, h and Supplementary Fig. 4). The degeneracy of phase tetragonality within mesoscale domains further suggests the scenario of PhT frustration occurred at the $x_{\mathrm{tcr}}^{\mathrm{T}}$. It is noteworthy that the structural anomaly is irrelevant to compositional segregation, which is evidenced by energy dispersive X-ray spectroscopy maps of the elements (Fig. 1i-l).

Decoupled polarization-strain relationship. Our statistical measurement shows that the average domain size is very small, $<d>\approx 5.3 \mathrm{~nm}$ in the PZT crystals (Supplementary Fig. 5).
According to the classic domain theory ${ }^{5}, d \propto \sqrt{E_{\mathrm{DW}}}\left(E_{\mathrm{DW}}\right.$ : domain wall energy), this suggests that the $E_{\mathrm{DW}}$ is very low, which monotonically decreases from ca. 250 to $38 \mathrm{~mJ} \mathrm{~m}^{-2}$, via a concave inflection point at $x_{\mathrm{tcr}}^{\mathrm{T}}$, with decreasing $x$ in terms of our calculation (Supplementary Figs. 6 and 7). Further, atomic-scale domain structures were acquired using the negative sphericalaberration imaging technique ${ }^{29}$. Owing to instability of ordered state to random fields $s^{30}$, the nanodomains form irregular configuration in the $x<x_{\mathrm{tcr}}^{\mathrm{T}}$ crystals $^{31}$ and the lattice tetragonality shows obvious fluctuation in real space. This is well manifested by a topological vortex structure, where the average lattice ratio for the $\mathrm{FE}_{\mathrm{T}}$ and $\mathrm{FE}_{\mathrm{M}}$ phase is measured as $(c / a)_{\mathrm{T}} \approx 1.036$ and $(c / a)_{\mathrm{M}} \approx 1.022$ (Fig. $2 \mathrm{a}, \mathrm{b}$ and Supplementary Fig. 8a-d). A unitcell-wise correlation of lattice ratio with polar displacement of oxygen $\left(\delta_{\mathrm{O} 2-\mathrm{Pb}}\right)$ is plotted for further data statistics and analysis (Fig. 2c). We find that the $c / a$ ratio and $\delta_{\mathrm{O} 2-\mathrm{Pb}}$ both follow a Gaussian-type distribution and separately peak at 1.028 (fullwidth at half-maximum, FWHM $=0.066$ ) and $22.9 \mathrm{pm}$. Together with individual phase analysis, this evidences a coupled polarization-strain relation, $P_{\mathrm{S}}^{2}=\sigma(\sigma=c / a-1)^{32}$, in the secondorder-transition crystals (Supplementary Fig. 8e, $\mathrm{f}$ ).

In the $x=0.65$ crystal, coexisting tetragonal and monoclinic phases and degeneracy of their lattice tetragonality are also confirmed by analysis on atomic-scale TEM images, e.g., the $c / a$ ratio histogram peaks at 1.012 for the $c$ domain and its FWHM is narrowed to 0.054 (Fig. $2 \mathrm{~d}-\mathrm{f}$ ). Specifically, we find that the polar displacement of oxygen $\left(\delta_{\mathrm{O} 2-\mathrm{Pb}}\right)$ exhibits a bimodal distribution, whose peaks locate around 16.1 and $32.4 \mathrm{pm}$, respectively.
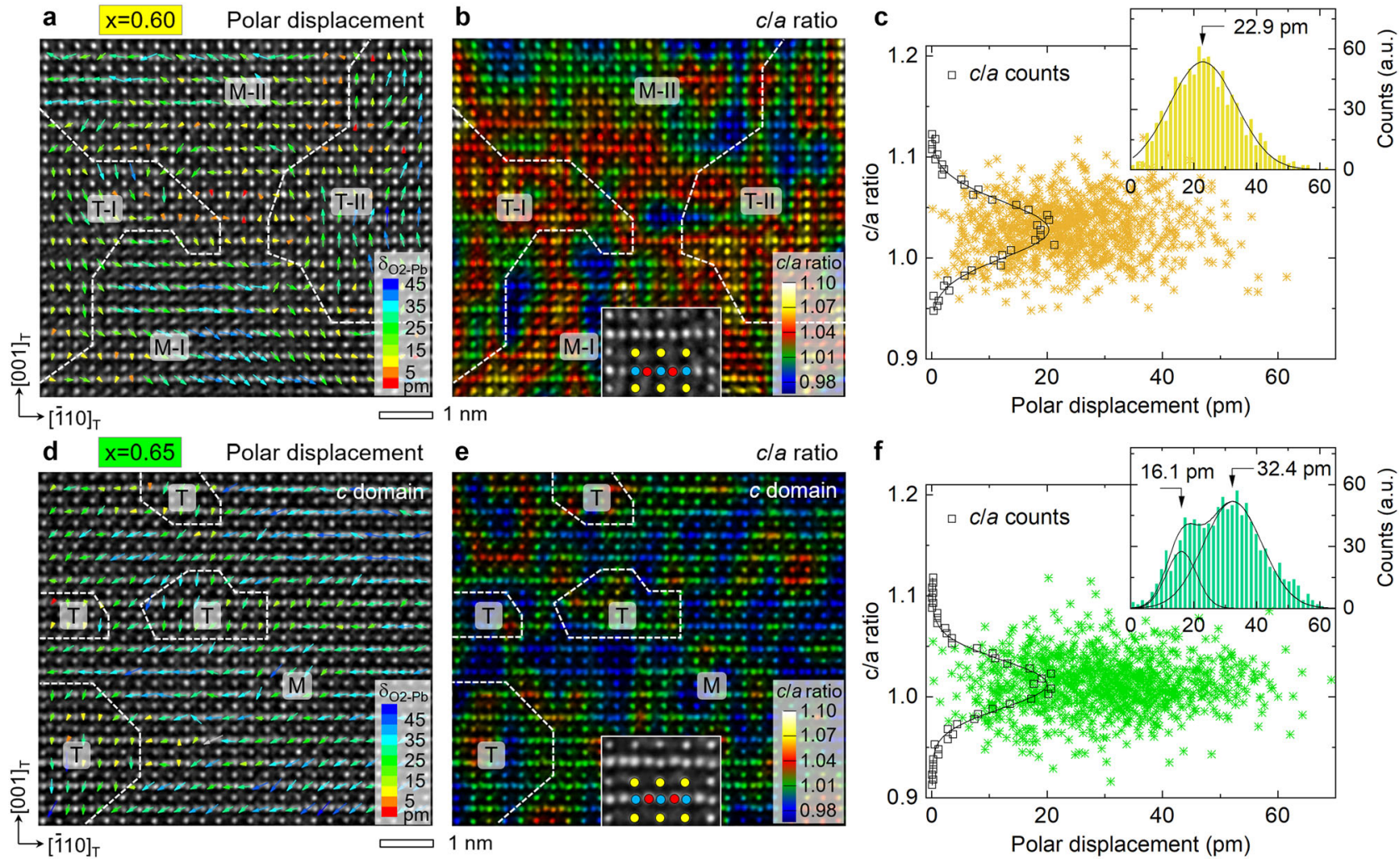

Fig. 2 Nanoscale phase coexistence and polarization-strain relation. a, d Atomic-resolution TEM image of $x=0.60$ and 0.65 crystal recorded along $[110]_{\mathrm{T}}$ direction and overlaid with a map of $\mathrm{O}_{2}$ column displacements (color arrows) relative to the centers of $\mathrm{Pb} / \mathrm{O} 1 \mathrm{columns}\left(\delta_{\mathrm{O} 2-\mathrm{Pb}}\right.$ ), respectively. $\mathbf{b}$, e Unit-cell-wise $c$ / a ratio map (after interpolation) overlaid on the atomic-resolution TEM image of $x=0.60$ and 0.65 crystal, respectively. The white dotted lines in (a, b) and (d, e) mark the tetragonal $(\mathrm{T}) /$ monoclinic $(\mathrm{M})$ phase boundaries. The atomic column types are denoted with $\mathrm{Pb} / \mathrm{O} 1-\mathrm{yellow}, \mathrm{Zr} / \mathrm{Ti}-$ blue, $\mathrm{O}_{2}$-red. c, $\mathbf{f}$ Unit-cell-wise correlation of $\mathrm{c} / \mathrm{a}$ ratio with $\mathrm{O}_{2}$ polar displacement for the $x=0.60$ and 0.65 crystal, respectively. The interior inset is a statistical profile of the $c / a$ ratio (squares) fitted by a Gaussian function (solid line) and the right-side inset is a corresponding statistical histogram of the polar displacement. 
Clearly, this evidences a decoupled $P_{\mathrm{S}} \sim \sigma$ relation in the tricritical ferroelectric, which is further supported by strongly charged but unstable ferroelastic domain walls if the coupling relation still holds (see "Methods"). On the first-order transition side $\left(x>x_{\mathrm{tcr}}^{\mathrm{T}}\right)$, the coupled $P_{\mathrm{S}} \sim \sigma$ relation is verified again by our quantitative measurement on atomically resolved tetragonal ferroelastic domains (Supplementary Fig. 9). These atomic-scale details further indicate a frustrated behavior of $\mathrm{PhT}$ at the tricritical point.

Abnormal critical exponents. To verify the frustration scenario at the $x_{\text {tcr }}^{\mathrm{T}}$, critical exponents were analyzed from temperature dependent dielectric constant $(\varepsilon)$ of the PZT crystals (Fig. 3a, b and Supplementary Fig. 10). By fitting $\varepsilon$ at $t>T_{\mathrm{C}}$ using a modified Curie-Weiss law, $1 / \varepsilon-1 / \varepsilon_{m}=\left(t-T_{0}\right)^{\gamma / C}\left(\varepsilon_{m}\right.$ : the maximum of $\varepsilon, C$ : Curie constant, $T_{0}$ : Curie-Weiss temperature), we find that the $x \leq x_{\mathrm{tcr}}^{\mathrm{T}} \mathrm{PZT}$ crystals are characteristic of a pronounced precursor behavior $^{33}$. This is manifested by a large gamma exponent $(\gamma>1)$ and deviation from the Curie-Weiss law due to formation of polar clusters at $t>T_{\mathrm{C}} 6,7,15$. A systematic change of $\gamma$ is unveiled by our effective Hamiltonian Monte Carlo simulations, which decreases from 2.26 to 1.17 with increasing $x$ at $x \leq x_{\mathrm{tcr}}^{\mathrm{T}}$, above which the classic mean-field value $(\gamma=1)$ is observed (Fig. 3c). In addition to this, Curie-constant ratio $\left(\left|C^{-} / C^{+}\right|\right)$is measured by fitting $\varepsilon$ below $\left(C^{-}\right)$and above $\left(C^{+}\right) T_{\mathrm{C}}$ using CurieWeiss law to testify the $\mathrm{PhT}$ orders according to the Landau-Devonshire theory ${ }^{5}$. Associated with a step-wise increase from 2 (second order) to 8 (first order) with $x$, the $\left|C^{-} / C^{+}\right|$ratios of $x=0.54$ and 0.60 crystals are found to be $3.45 \pm 0.19$ and $3.83 \pm 0.26$, respectively. This ratio is measured as $\left|C^{-} / C^{+}\right| \approx 5.4$ for the $x=0.65$ crystal (Fig. $3 \mathrm{~d}$ ), which differs from the meanfield value of 4 at the tricritical point ${ }^{34}$.

The critical exponent anomalies verify the existence of a frustrated $\mathrm{PhT}$ order at the $x_{\mathrm{tcr}}^{\mathrm{T}}$ (Fig. 3e). Given the nearly coincident loss tangent $(\tan \delta)$ of the $x<x_{\mathrm{tcr}}^{\mathrm{T}}$ crystals, the discernible thermal hysteresis at $T_{\mathrm{C}}$ of $\mathrm{x}=0.65$ crystal further hints an anomaly of the PhT (Fig. 3a, b). To find out origin of the frustration, the precursor dynamics reflecting optic-acoustic mode-mode coupling at $t>T_{\mathrm{C}}$ was probed by analyzing local $\gamma$ exponent within a restricted temperature interval $(\Delta t=10 \mathrm{~K})^{35}$. For the $x=0.65$ crystal, two localized polar glassy states featured by gamma $(\gamma)$ peaks were identified at $T_{g 1}=t-T_{\mathrm{C}} \approx 40 \mathrm{~K}$ and $T_{g 2}=t-T_{C} \approx 63 \mathrm{~K}$, respectively (Fig. $3 \mathrm{f}$ and Supplementary Fig. 11). Around $T_{g 1}$, the gamma has a peak value of $\gamma \approx 1.25$ in both heating- and cooling-cycle profiles, while the peak value at $T_{g 2}$ decreases from $\sim 1.46$ in the heating cycle to $\sim 1.30$ in the cooling cycle. Critical behavior study indicates that corresponding to the characteristic $\gamma$ value, the polar glass states adopt shortrange $3 \mathrm{D}$-Ising $(\gamma \approx 1.25)$ and long-range 3D-random-Ising $(\gamma \approx 1.46)$ universality classes $^{6}$, respectively. This reveals that accompanied with heating- and cooling-dependent structural changes, the frustrated PhT order may result from an intricate interplay of competing short-range dipolar orders with spontaneously developed long-range ones ${ }^{36}$ around $t T_{\mathrm{C}}$. Despite the $\gamma$ exponent being large $(1.22 \leq y \leq 1.44)$ in the $x<x_{\text {tcr }}^{\mathrm{T}}$ crystals as well, a similar competition is not established due to presence of single $\gamma$ peak around either $T_{g 1}$ or $T_{g 2}$ in the heating or cooling

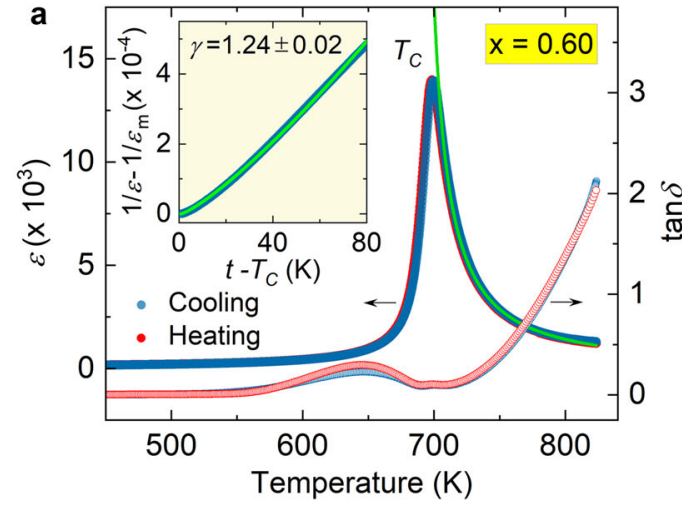

b

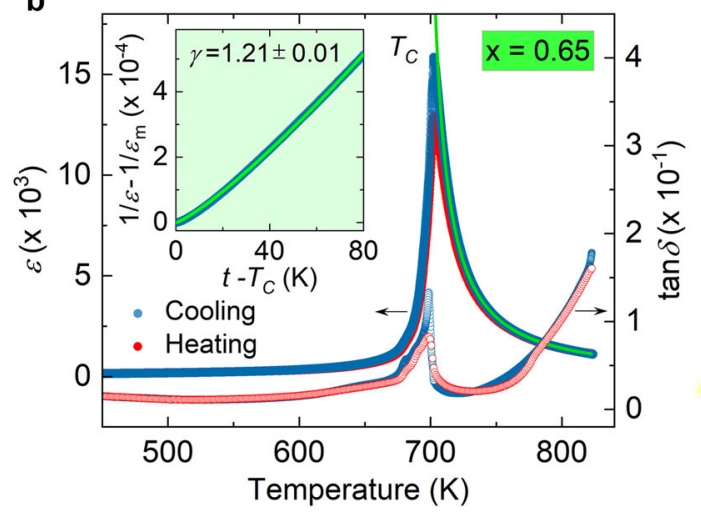

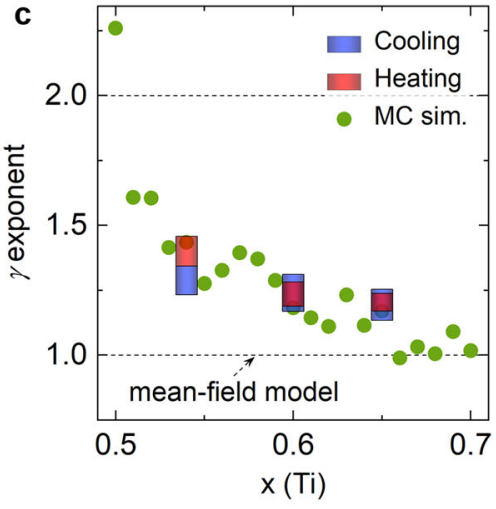

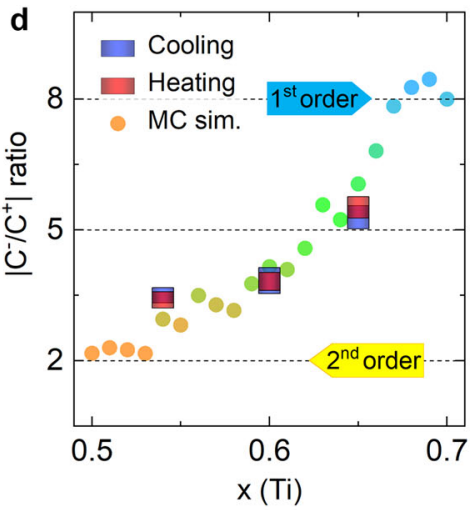

e

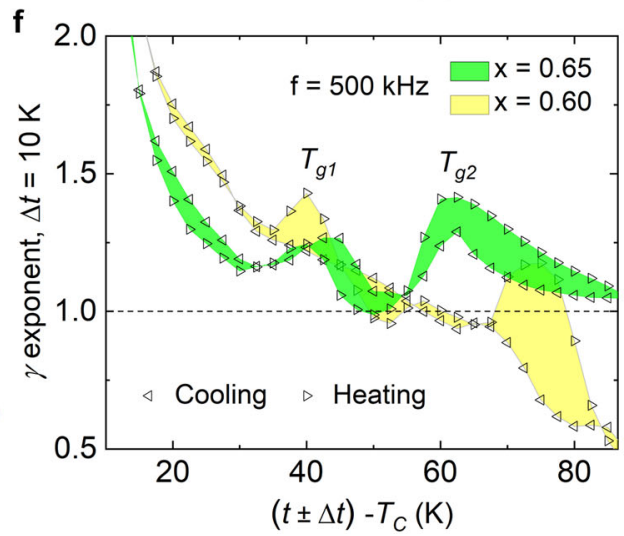

Fig. 3 Dielectric constants and critical exponents. a, b Temperature-dependent $\varepsilon$ for $x=0.60\left(T_{\mathrm{C}} \approx 699 \mathrm{~K}\right)$ and $0.65\left(T_{\mathrm{C}} \approx 703 \mathrm{~K}\right)$ crystal measured at $f=500 \mathrm{kHz}$, respectively. The green solid lines are fitting to the cooling $\varepsilon$ data at $t>T_{\mathrm{C}}$ using a modified Curie-Weiss law $1 / \varepsilon-1 / \varepsilon_{m}=\left(t-T_{0}\right) \gamma / C$. c, $\mathbf{d}$ Evolution of $\gamma$ exponent and Curie-constant ratio $\left|\mathrm{C}^{-} / \mathrm{C}^{+}\right|$from effective Hamiltonian Monte Carlo simulations and their experimental values measured at $f=500,333$, and $100 \mathrm{kHz}$, respectively. e lllustration of the frustrated PhT order, with $\left|\mathrm{C}^{-} / \mathrm{C}^{+}\right| \approx 5$ and $\gamma>1$, arising from competing first- and second-order transitions at $x_{\mathrm{tcr}}^{\top}$ and $T_{\mathrm{C}}$. f Temperature-dependent $\gamma$ exponent measured within a restricted temperature interval $(\Delta t=10 \mathrm{~K})$ at $t>T_{\mathrm{C}}$ and $f=500 \mathrm{kHz}$ for $x=0.65 \mathrm{and} 0.60 \mathrm{crystal}$ (see Supplementary Fig. 11 for $x=0.54$ case). 

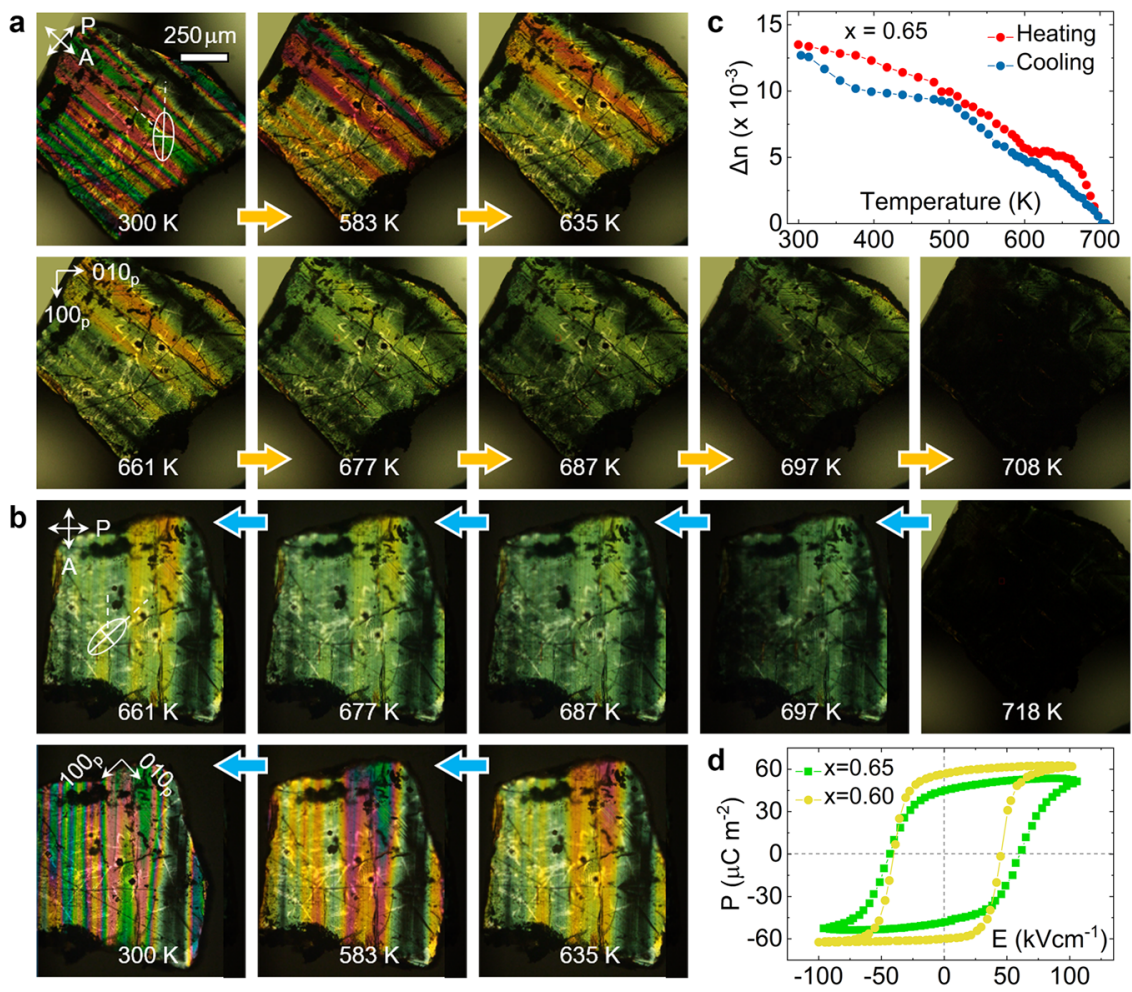

Fig. 4 Evidence of competing first- and second-order transitions at $\boldsymbol{x}_{\mathbf{t c r}}^{\mathbf{T}}$ around $\boldsymbol{T}_{\mathbf{c}}$ and ferroelectric anomaly. $\mathbf{a}$, $\mathbf{b}$ Temperature-dependent ferroelastic domain structure evolution in a pseudocubic $(001)_{p}$ platelet of the $x=0.65$ PZT crystal observed during heating and cooling cycles by PLM, respectively. The polarizer $(P)$, analyzer $(A)$, and optical indicatrix are denoted on the image with respect to the crystallographic orientation. $\mathbf{c}$ Temperature-dependent birefringence data of the $x=0.65$ PZT crystal. $\mathbf{d} P-E$ hysteresis loops of the $x=0.60$ and 0.65 PZT crystals measured at room temperature with frequency $f=10 \mathrm{~Hz}$.

cycle. Additionally, we also notice that the $\tan \delta$ in $x<x_{\text {tcr }}^{\mathrm{T}}$ crystals is one order of magnitude higher than that in the $x=0.65$ crystal, which can be attributed to the formation of conductive channels due to random arrangement of nanodomains ${ }^{31,37,38}$.

Birefringent evidence of PhT frustration. Being consistent with the precursor dynamics identified at $t>T_{\mathrm{C}}$, our variabletemperature polarized light microscopy (PLM) experiments directly reveal the competion of $\mathrm{PhT}$ orders at $t \leq T_{\mathrm{C}}$. From heating-cycle PLM snapshots, we see that the mesoscale band-like ferroelastic domain array is well preserved until $t \approx T_{\mathrm{C}}$, at which the domain width and wall position start to evolve dynamically (Fig. 4a). Preservation of the long-range dipolar correlation indicates that the first-order transition overbears the secondorder one ${ }^{39}$, which is evidenced by an abrupt drop of birefringence $(\Delta n)$ as $t$ approaching $T_{\mathrm{C}}$ (Fig. 4c). However, the formation of band-like ferroelastic domains is much delayed in the cooling cycle (Fig. 4b), which can be identified from the domain-contrast change at the identical $t$ points ranging from 697 to $635 \mathrm{~K}$. The enhanced short-range dipolar correlation suggests that the second-order transition prevails over the first-order on $e^{40}$, as proved by a smooth transition of $\Delta n$ around $T_{\mathrm{C}}$ (Fig. 4c). On the physical property aspect, the $\mathrm{PhT}$ frustration is also manifested by abnormal ferroelectric property at room temperature (Fig. 4d). With respect to the symmetric polarization-electric field $(P-E)$ loops of PZT undergoing the first- ${ }^{41}$ and second-order transitions, we find that the remnant polarization is reduced by $\sim 20.4 \%$ relative to the $x<x_{\text {tcr }}^{\mathrm{T}}$ PZT crystals. Furthermore, a very large built-in field $\left(\Delta E_{\mathrm{C}} \approx 20 \mathrm{kV} \mathrm{cm}^{-1}\right)$, an order of magnitude higher than that of $x<x_{\text {tcr }}^{\mathrm{T}}$ crystals, is observed in the tricritical ferroelectric.
Monte Carlo simulations. To further verify the experimental results, we performed effective Hamiltonian Monte Carlo simulations on $80 \times 80 \times 5$ supercells of PZT near $x_{\text {tcr }}^{\mathrm{T}}$ at room temperature, in which only tetragonal ferroelastic $c$ and $a$ domains with equivalent volume, identical dipolar magnitude, and orientation along $[001]_{\mathrm{T}}$ are configured at the initial state. After relaxation for 40,000 Monte Carlo sweeps, we find that the initial $c$ and $a$ domains in the second-order-transition PZT $\left(x<x_{\text {tcr }}^{\mathrm{T}}\right)$ supercells disappear and the entire structure transforms into coexisting tetragonal or monoclinic nanodomains, whose characteristic size is several nanometers (Fig. 5a). In good agreement with the experimental result, the hierarchical domain structure is nicely reproduced in frustrated-order-transition PZT supercells with the Ti concentration around $x_{\mathrm{tcr}}^{\mathrm{T}}$. Meanwhile, the nanodomain sizes are found to increase gradually with decreasing $x$ owing to enhanced flexibility in the orientation of local dipoles (Fig. 5b). On the first-order-transition $\left(x>x_{\mathrm{tcr}}^{\mathrm{T}}\right)$ side, the ferroelastic $90^{\circ}$ domains are preserved due to dominance of the tetragonal phase, albeit with unequal width for the $c$ and $a$ domains (Fig. 5c). In addition, the $P_{\mathrm{S}} \sim \sigma$ coupling and decoupling behaviors across the $x_{\mathrm{tcr}}^{\mathrm{T}}$ are also confirmed in our Monte Carlo simulations (Fig. 5d, e and Supplementary Fig. 12).

Discussion. Besides the evidences of the critical exponent and birefringence data, the $\mathrm{PhT}$ frustration at $x_{\mathrm{tcr}}^{\mathrm{T}}$ can also be understood from the lattice distortion point of view ${ }^{36}$. With respect to the $\mathrm{PE}_{\mathrm{C}}$ phase, freezing of the glassy states at $t<T_{\mathrm{C}}$ gives rise to locally favored tetragonal and monoclinic ferroelectric orders, which couple with their individual lattice symmetry ${ }^{31}$. However, as being subject to the long-range lattice 

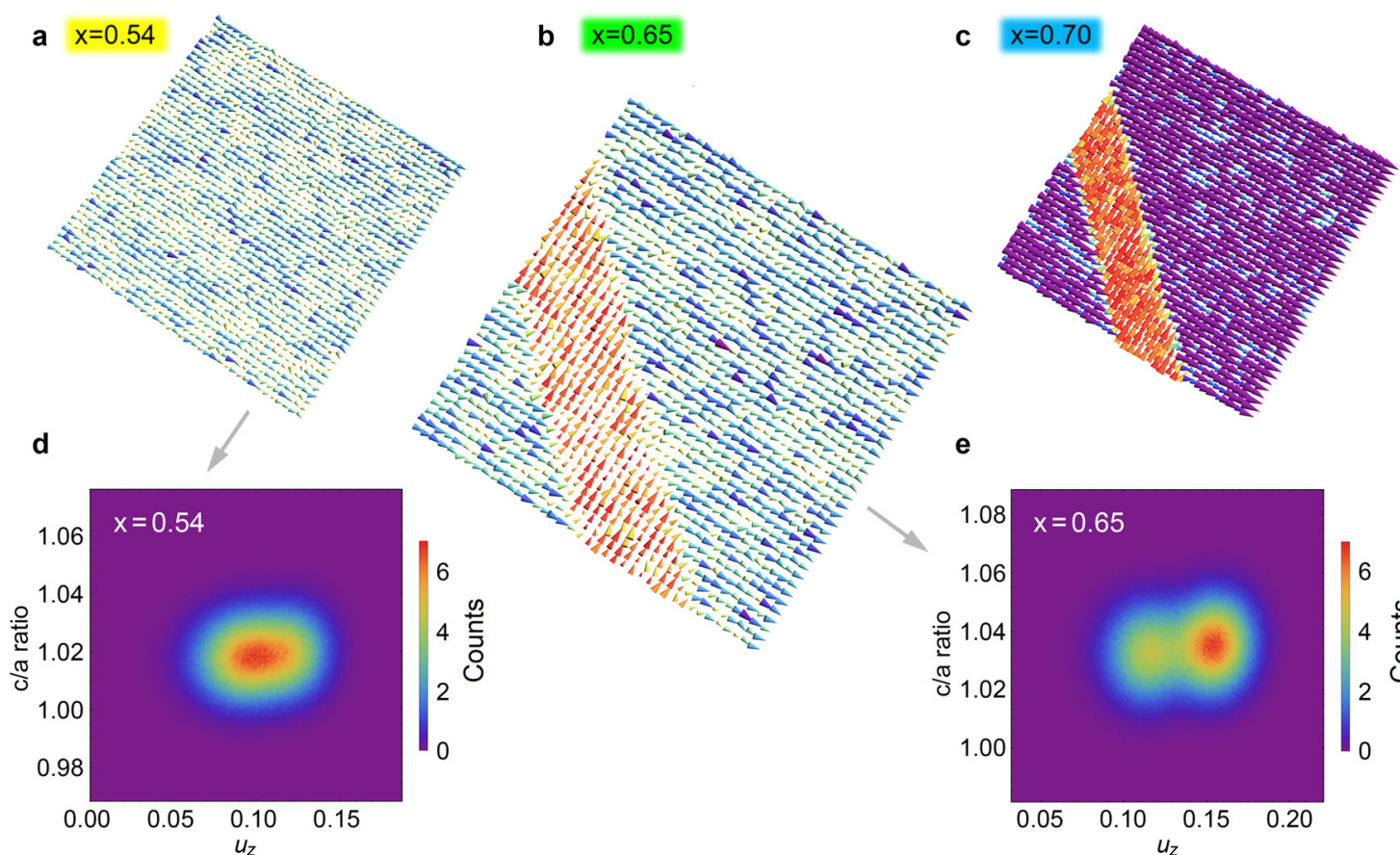

e

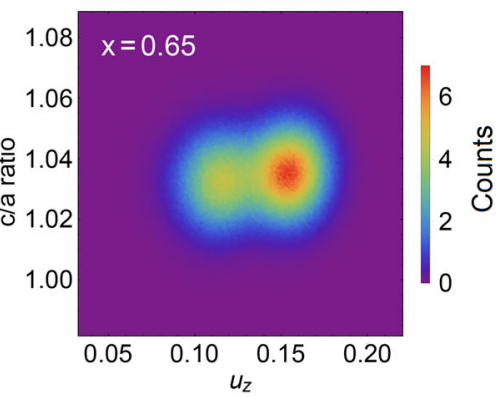

Fig. 5 Monte Carlo simulated domain structure evolution near $\boldsymbol{x}_{\mathbf{t c r}}^{\boldsymbol{\top}}$ at room temperature. a Nesting tetragonal and monoclinic nanodomains induced by second-order transition in $x<x_{\text {tcr }}^{\top}$ PZT. $\mathbf{b}$ Hierarchical domain structure induced by frustrated-order transition in $x=x_{\text {tcr }}^{\top} \mathrm{PZT}$. $\mathbf{c}$ Ferroelastic $90^{\circ}$ domain structure induced by first-order transition in $x>x_{t c r}^{\top} \mathrm{PZT}$. The local electric dipoles are colored according to their projection on the [010] $]_{p}$ direction while the arrowhead size indicates the magnitude of the electric dipole. The supercells ( $40 \times 40 \times 5$ pseudocubic unit cells) are viewed along the [001] direction. d, e Joint probability distribution of local mode $\left(u_{z}\right)$ and local tetragonality for $\mathbf{d} x=0.54$ and $\mathbf{e}$ either $c$ or $a$ domain of $x=0.65$ PZT supercells, respectively. The colors denote probability distribution in the $2 \mathrm{D}$ correlation map.

deformation, the short-range polar orders compete within the mesoscale domains and fail to simultaneously couple with the uniformly distorted lattices. This leads to geometrically frustrated structural features, e.g., the decoupled $P_{\mathrm{S}} \sim \sigma$ relation, which can be attributed to the "slower breathing" of dipolar inhomogeneity according to first-principle-based simulations ${ }^{42}$. Accordingly, the greatly diminished polarization anisotropy (see Supplementary Figs. 6 and 7) becomes responsible for the configurational change of ferroelastic domains under varied boundary conditions ${ }^{12}$. Therefore, analogous to the spin and charge frustration observed in ferroic materials ${ }^{13,43}$, the $\mathrm{PhT}$ frustration gives rise to an ambiguous PhT order at the tricritical composition point. It is noteworthy that the frustrated transition order is distinct from the coexistence or mixture of first order with second-order transitions ${ }^{44}$, which can easily be identified from the correlation of $\mathrm{PhT}$ order with the corresponding structural feature.

Regarding the critical exponent $y$ that is larger than one, it reflects the effective dimensionality of the order parameter and the interactions at play, rather than any disorder or frustration. To verify the existence of geometric frustration, incompatible fundamental interplay of a physical quantity with the lattice geometry and ground state degeneracy should be fulfilled simultaneously $6,13,16,43$. In our Monte Carlo simulations, we find that the tricritical transition takes place in a composition interval of $\Delta x_{\mathrm{tcr}}^{\mathrm{T}} \approx 0.10$, which is denoted by dashed lines in Fig. 1e. For ferroic and superconducting systems with apparent tricritical points $4,8,34,45$, one may foresee that the $\mathrm{PhT}$ frustration occurs as the tricritical point is accessed by tuning composition, electric, magnetic field, and pressure. For systems with hidden tricritical points, e.g., $\mathrm{BaTiO}_{3}$, the frustration scenario probably validates as well given the simultaneous presence of critical exponent anomaly $(\gamma \approx 1.08)^{7}$, structural hierarchy and degenerate lattice ratio for the coexisting tetragonal and monoclinic phases ${ }^{46,47}$.
This even applies to the thermotropic phase boundary ${ }^{47}$, where the first-order ferroelectric transition competes with the excited second-order transition, which is evidenced by presence of lowsymmetric structural phases ${ }^{48}$.

In summary, we report a frustrated PhT order at the tricritical point of ferroelectric PZT solid solution. Given the ubiquitous tricritical point, our findings suggest a generic mechanism to decipher PhT-related unusual critical phenomena in ferroic materials, which are featured by abnormal critical exponents, structural hierarchy, degenerate lattice tetragonality for coexisting phases, and may possibly be extended to ferroelectrics with diffuse PhTs near the MPBs ${ }^{7,49}$. Particularly, the frustrated PhT scenario offers an important degree of freedom to engineer hierarchical domains, which has been reported to play important roles in improving material performances such as piezoelectricity ${ }^{50}$, magnetoelectric effect ${ }^{3}$, shape memory ${ }^{51}$, and electrostatic energy storage $^{52}$. Therefore, it is believed that this work may inspire extensive research interest on exploring PhT-related frustrated states and design of material functionality in a more flexible way.

\section{Methods}

Materials preparation. Lead zirconate titanate PZT $(x=0.54,0.60$, and 0.65$)$ single crystals were grown by a top-seeded solution growth technique ${ }^{53}$, and the PZT $(x=0.90)$ thin films were grown by pulsed laser deposition ${ }^{54,55}$. PLM (Olympus BX60) was used to characterize temperature-dependent domain evolution data. FEI Helios NanoLab 400s focused ion beam (FIB) system was used for preparing the lamella specimens, before which an Au layer (thickness $\sim 25 \mathrm{~nm}$ ) was coated on the sample surfaces. To protect the samples from being damaged by Ga ions, electron-induced carbon $(\sim 180 \mathrm{~nm})$ and ion-induced Pt $(\sim 4 \mu \mathrm{m})$ layers were deposited on the region of interests (area $\sim 20 \mu \mathrm{m} \times 2 \mu \mathrm{m}$ ). After taking out the lamella by making trenches on both sides, the lamella was welded to the TEM grids, milled at $30 \mathrm{kV}$ with $2.8 \mathrm{nA}-93 \mathrm{pA}$ currents and followed by a final cleaning at $5 \mathrm{kV}$ and $47 \mathrm{pA}$. To remove the surface contamination and damage layer, the NanoMill Model 1040 system operated at $500 \mathrm{~V}$ was used to further clean and thin down the lamella specimens. 
Electron microscopy imaging experiment. The dark-field imaging and SAED experiments were performed on an FEI Tecnai F20 microscope operated at $200 \mathrm{kV}$. Referring to the $\mathrm{SrTiO}_{3}$ standard, the lattice ratios of PZT crystals were measured in a quantitative way from the SAED patterns. The atomic-resolution TEM and 4D-STEM experiments were performed on image- and probe-corrected FEI Titan 80-300 microscopes operated at $300 \mathrm{kV}$, respectively. By fitting atomic column peak intensities using 2D-Gaussian-function-based maximum likelihood estimation ${ }^{56,57}$, we simulated the atomic-resolution images using CrystalKit-MacTempas software package. An FEI Titan 80-200 ChemiSTEM microscope, equipped with a Super-X energy dispersive X-ray spectrometer, was used for compositional analysis.

Estimation of bound charges at ferroelectric domain wall. Supposing the $P_{\mathrm{S}}^{2}=\sigma$ relation holds in the $x=0.65$ crystal, referring to $\mathrm{PbTiO}_{3}$ standard ${ }^{54}\left(P_{\mathrm{S}}=96.8 \mu \mathrm{C} \mathrm{cm}^{-2}\right.$, $c / a=1.0643$ ), the coupling coefficient is determined as $\kappa=145,727 \mu \mathrm{C}^{2} \mathrm{~cm}^{-4}$. For the ferroelastic domains with $(c / a)_{a}=1.044$ and $(c / a)_{c}=1.014$, our calculation reveals that the density of bound charges is $\Delta P_{\mathrm{S} \perp}=24.7 \mu \mathrm{C} \mathrm{cm}^{-2}$ normal to the ferroelastic wall plane, which is equal to the $P_{\mathrm{S}}$ of $\mathrm{BaTiO}_{3}{ }^{58}$. To lower energy of the strongly charged wall structures, an especially high density of free carriers, beyond the available limit of the material itself ${ }^{59}$, is needed. To minimize the electrostatic energy, the charged domain walls usually show curved morphology ${ }^{60}$. This is also different from our experimental observations. These results therefore alternatively refute the $P_{\mathrm{S}} \sim \sigma$ coupling relation in the $x=0.65$ crystal.

Dielectric and ferroelectric property measurement. The dielectric properties of PZT single crystals were measured using a Novocontrol Alpha high-resolution broadband dielectric spectrometer. The dimensions of the crystals, oriented along $(001)_{p},(011)_{p}$, and $(001)_{p}$, used for the measurement are $1.4 \times 0.8 \times 0.162$, $1.5 \times 1.67 \times 0.05$, and $1.9 \times 1.2 \times 0.22 \mathrm{~mm}^{3}$ for the $x=0.54,0.60$, and 0.65 crystal, respectively. Typically, a small signal ac electric field $\left(1 V_{\mathrm{rms}}\right)$ was applied for the standard dielectric spectroscopy measurements. The Curie-constant ratio was measured beside $T_{\mathrm{C}}$ in a temperature interval of $10-20 \mathrm{~K}$. A standardized ferroelectric analyzer system (TF Analyzer 2000; aixACCT, Germany) was used to measure the ferroelectric property at room temperature.

Monte Carlo simulations. Monte Carlo simulations on PZT bulks were performed using an ab initio-based effective Hamiltonian model ${ }^{61,62}$, which parametrizes the Born-Oppenheimer energy landscape in terms of local modes about B-site polar displacement, oxygen octahedral rotation, and takes into account acoustic phonon branches parameterized through homogeneous strain tensor and A-site displacement within each unit cell. The alloying effects are mimicked by introducing local fields through breaking local cubic symmetry in the PE state via compositional disorder and different values of on-site coefficients of the effective Hamiltonian. The supercell lateral sizes were chosen to be of $12 \times 12 \times 12$ or $80 \times 80 \times 5$ unit cells along the pseudocubic $(\mathrm{p})[100]_{\mathrm{p}},[010]_{\mathrm{p}}$, and $[001]_{\mathrm{p}}$ axis, respectively. The former and latter geometry was used to obtain temperature-dependent macroscopic property and domain structure, respectively. To compute equilibrium property for each considered composition, we firstly performed temperature annealing simulations to obtain the raw values of $T_{\mathrm{C}}$, where the system was cooled from $1500 \mathrm{~K}$ down to $100 \mathrm{~K}$ with a step size of $50 \mathrm{~K}$. For $x=54,60,65$, and 100 , the annealing simulations were then repeated under hydrostatic pressure varying from -5 to $5 \mathrm{GPa}$ with an increment of $0.1 \mathrm{GPa}$. This helps to define the effective pressure and to calibrate the simulated $T_{\mathrm{C}}$ with respect to the experimental one. By interpolating the effective pressure values, we obtain the external pressure for all considered compositions in the range of $x=0.50-0.70$. This procedure allows to correct for errors induced by LDA approximation while constructing the effective Hamiltonian model, and the errors related to the absence of anharmonic elastic contributions that are responsible for thermal lattice expansion.

\section{Data availability}

The authors declare that all data supporting the findings of this study are available within the paper and its Supplementary information files.

\section{Code availability}

The codes used in this study are available from the corresponding authors upon reasonable request.

Received: 26 January 2021; Accepted: 18 August 2021; Published online: 07 September 2021

\section{References}

1. Kutnjak, Z., Petzelt, J. \& Blinc, R. The giant electromechanical response in ferroelectric relaxors as a critical phenomenon. Nature 441, 956-959 (2006).

2. Liu, W. \& Ren, X. Large piezoelectric effect in $\mathrm{Pb}$-free ceramics. Phys. Rev. Lett. 103, 257602 (2009)
3. Evans, D. M. et al. Magnetic switching of ferroelectric domains at room temperature in multiferroic PZTFT. Nat. Commun. 4, 1534 (2013).

4. Bianchi, A. et al. First-order superconducting phase transition in CeCoIn5. Phys. Rev. Lett. 89, 137002 (2002).

5. Lines, M. E. \& Glass, A. M. Principles and Applications of Ferroelectrics and Related Materials (Oxford Univ. Press, 2001).

6. Almahmoud, E., Kornev, I. \& Bellaiche, L. Critical behavior in ferroelectrics from first principles. Phys. Rev. Lett. 102, 105701 (2009).

7. Uchino, K. \& Nomura, S. Critical exponents of the dielectric constants in diffused-phase-transition crystals. Ferroelectrics 44, 55-61 (1982).

8. Kaluarachchi, U. S., Bud'ko, S. L., Canfield, P. C. \& Taufour, V. Tricritical wings and modulated magnetic phases in $\mathrm{LaCrGe}_{3}$ under pressure. Nat. Commun. 8, 546 (2017).

9. Wei, X. K. et al. An unconventional transient phase with cycloidal order of polarization in energy-storage antiferroelectric $\mathrm{PbZrO}_{3}$. Adv. Mater. 32, 1907208 (2020).

10. Gleiser, M. Dynamics of weak first order phase transitions. Phys. Rev. Lett. 73, 3495-3498 (1994).

11. Zhang, Q., Cagin, T. \& Goddard, W. A. III The ferroelectric and cubic phases in $\mathrm{BaTiO}_{3}$ ferroelectrics are also antiferroelectric. Proc. Natl Acad. Sci. USA 103, 14695-14700 (2006)

12. Rossetti, G. A., Khachaturyan, A. G., Akcay, G. \& Ni, Y. Ferroelectric solid solutions with morphotropic boundaries: vanishing polarization anisotropy, adaptive, polar glass, and two-phase states. J. Appl. Phys. 103, 114113 (2008)

13. Balents, L. Spin liquids in frustrated magnets. Nature 464, 199-208 (2010).

14. Shintani, H. \& Tanaka, H. Frustration on the way to crystallization in glass. Nat. Phys. 2, 200-206 (2006).

15. Choudhury, N., Walizer, L., Lisenkov, S. \& Bellaiche, L. Geometric frustration in compositionally modulated ferroelectrics. Nature 470, 513-517 (2011).

16. Nahas, Y., Prokhorenko, S. \& Bellaiche, L. Frustration and self-ordering of topological defects in ferroelectrics. Phys. Rev. Lett. 116, 117603 (2016).

17. Porta, M. \& Lookman, T. Effects of tricritical points and morphotropic phase boundaries on the piezoelectric properties of ferroelectrics. Phys. Rev. B 83, 174108 (2011).

18. Whatmore, R. W., Clarke, R. \& Glazer, A. M. Tricritical behaviour in $\mathrm{PbZr}_{\mathrm{x}} \mathrm{Ti}_{1-\mathrm{x}} \mathrm{O}_{3}$ solid solutions. J. Phys. C Solid State Phys. 11, 3089 (1978).

19. Solanki, R. S., Mishra, S. K., Kuroiwa, Y., Moriyoshi, C. \& Pandey, D. Evidence for a tricritical point coinciding with the triple point in $\left(\mathrm{Pb}_{0.94} \mathrm{Sr}_{0.06}\right)\left(\mathrm{Zr}_{\mathrm{x}} \mathrm{Ti}_{1-\mathrm{x}}\right)$ $\mathrm{O}_{3}$ : a combined synchrotron $\mathrm{x}$-ray diffraction, dielectric, and Landau theory study. Phys. Rev. B 88, 184109 (2013).

20. Zhang, N. et al. The missing boundary in the phase diagram of $\mathrm{PbZr}_{1-\mathrm{x}} \mathrm{Ti}_{\mathrm{x}} \mathrm{O}_{3}$ Nat. Commun. 5, 5231 (2014)

21. Rossetti, G. A. \& Navrotsky, A. Calorimetric investigation of tricritical behavior in tetragonal $\mathrm{Pb}\left(\mathrm{Zr}_{\mathrm{x}} \mathrm{Ti}_{1-\mathrm{x}}\right) \mathrm{O}_{3}$. J. Solid State Chem. 144, 188 (1999).

22. Asada, T. \& Koyama, Y. Ferroelectric domain structures around the morphotropic phase boundary of the piezoelectric material $\mathrm{PbZr}_{1-\mathrm{x}} \mathrm{Ti}_{\mathrm{x}} \mathrm{O}_{3}$. Phys. Rev. B 75, 214111 (2007).

23. Lehnen, P., Dec, J. \& Kleemann, W. Ferroelectric domain structures of $\mathrm{PbTiO}_{3}$ studied by scanning force microscopy. J. Phys. D Appl. Phys. 33, 1932-1936 (2000).

24. Remeika, J. P. \& Glass, A. M. The growth and ferroelectric properties of high resistivity single crystals of lead titanate. Mater. Res. Bull. 5, 37-45 (1970).

25. Zhang, N., Yokota, H., Glazer, A. M. \& Thomas, P. A. Neutron powder diffraction refinement of $\mathrm{PbZr}_{1-\mathrm{x}} \mathrm{Ti}_{\mathrm{x}} \mathrm{O}_{3}$. Acta Cryst. B 67, 386-398 (2011).

26. Frantti, J. et al. Phase transitions of $\mathrm{Pb}\left(\mathrm{Zr}_{\mathrm{x}} \mathrm{Ti}_{1-\mathrm{x}}\right) \mathrm{O}_{3}$ ceramics. Phys. Rev. B 66, 064108 (2002)

27. Soares, M. R., Senos, A. M. R. \& Mantas, P. Q. Phase coexistence region and dielectric properties of PZT ceramics. J. Eur. Ceram. Soc. 20, 321-334 (2000).

28. Noheda, B. et al. Tetragonal-to-monoclinic phase transition in a ferroelectric perovskite: the structure of $\mathrm{PbZr}_{0.52} \mathrm{Ti}_{0.48} \mathrm{O}_{3}$. Phys. Rev. B 61, 8687 (2000).

29. Jia, C. L., Lentzen, M. \& Urban, K. Atomic-resolution imaging of oxygen in perovskite ceramics. Science 299, 870-873 (2003).

30. Imry, Y. \& Ma, S.-K. Random-field instability of the ordered state of continuous symmetry. Phys. Rev. Lett. 35, 1399-1401 (1975).

31. Wei, X.-K. et al. Néel-like domain walls in ferroelectric $\mathrm{Pb}(\mathrm{Zr}, \mathrm{Ti}) \mathrm{O}_{3}$ single crystals. Nat. Commun. 7, 12385 (2016).

32. Qi, T., Grinberg, I. \& Rappe, A. M. Correlations between tetragonality, polarization, and ionic displacement in $\mathrm{PbTiO}_{3}$-derived ferroelectric perovskite solid solutions. Phys. Rev. B 82, 134113 (2010).

33. Bussmann-Holder, A., Beige, H. \& Völkel, G. Precursor effects, broken local symmetry, and coexistence of order-disorder and displacive dynamics in perovskite ferroelectrics. Phys. Rev. B 79, 184111 (2009).

34. Clarke, R. \& Benguigui, L. The tricritical point in $\mathrm{BaTiO}_{3}$. J. Phys. C Solid State Phys. 10, 1963 (1977).

35. Kleemann, W. et al. Uniaxial relaxor ferroelectrics: the ferroic random-field Ising model materialized at last. Europhys. Lett. 57, 14-19 (2002).

36. Carpenter, M. A., Salje, E. K. H. \& Graeme-Barber, A. Spontaneous strain as a determinant of thermodynamic properties for phase transitions in minerals. Eur. J. Mineral. 10, 21-691 (1998). 
37. Balke, N. et al. Enhanced electric conductivity at ferroelectric vortex cores in $\mathrm{BiFeO}_{3}$. Nat. Phys. 8, 81-88 (2011).

38. Wei, X. K., Jia, C. L., Roleder, K., Dunin-Borkowski, R. E. \& Mayer, J. In situ observation of point-defect-induced unit-cell-wise energy storage pathway in antiferroelectric $\mathrm{PbZrO}_{3}$. Adv. Funct. Mater. 31, 2008609 (2021).

39. An, Z. et al. Ferroelastic domain hierarchy in the intermediate state of $\mathrm{PbZr}_{0.98} \mathrm{Ti}_{0.02} \mathrm{O}_{3}$ single crystal. APL Mater. 9, 030702 (2021).

40. Wang, B., Wu, X., Ren, W. \& Ye, Z. G. Structure and properties of $\mathrm{Bi}\left(\mathrm{Zn}_{0.5} \mathrm{Ti}_{0.5}\right) \mathrm{O}_{3}-\mathrm{Pb}\left(\mathrm{Zr}_{1-\mathrm{x}} \mathrm{Ti}_{\mathrm{x}}\right) \mathrm{O}_{3}$ ferroelectric single crystals grown by a topseeded solution growth technique. IEEE Trans. Ultrason. Ferroelectr. Freq. Control 62, 1016-1021 (2015).

41. Karapuzha, A. S., James, N. K., Khanbareh, H., van der Zwaag, S. \& Groen, W. A. Structure, dielectric and piezoelectric properties of donor doped PZT ceramics across the phase diagram. Ferroelectrics 504, 160-171 (2016).

42. Ponomareva, I. \& Bellaiche, L. Nature of dynamical coupling between polarization and strain in nanoscale ferroelectrics from first principles. Phys. Rev. Lett. 101, 197602 (2008)

43. Ikeda, N. et al. Ferroelectricity from iron valence ordering in the chargefrustrated system $\mathrm{LuFe}_{2} \mathrm{O}_{4}$. Nature 436, 1136-1138 (2005).

44. Post, K. W. et al. Coexisting first- and second-order electronic phase transitions in a correlated oxide. Nat. Phys. 14, 1056-1061 (2018).

45. Cerro, J., del, Romero, F. J., Gallardo, M. C., Hayward, S. A. \& Jimenez, J. Latent heat measurement near a tricritical point: a study of the $\mathrm{KMnF}_{3}$ ferroelastic crystal. Thermochim. Acta 343, 89-97 (2000).

46. Kalyani, A. K. et al. Metastable monoclinic and orthorhombic phases and electric field induced irreversible phase transformation at room temperature in the lead-free classical ferroelectric $\mathrm{BaTiO}_{3}$. Phys. Rev. B 91, 104104 (2015).

47. Lummen, T. T. et al. Thermotropic phase boundaries in classic ferroelectrics. Nat. Commun. 5, 3172 (2014).

48. Vanderbilt, D. \& Cohen, M. Monoclinic and triclinic phases in higher-order Devonshire theory. Phys. Rev. B 63, 094108 (2001).

49. Otonicar, $\mathrm{M}$. et al. Multiscale field-induced structure of $(1-\mathrm{x}) \mathrm{Pb}\left(\mathrm{Mg}_{1 / 3} \mathrm{Nb}_{2 / 3}\right)$ $\mathrm{O}_{3-\mathrm{x}} \mathrm{PbTiOO}_{3}$ ceramics from combined techniques. Acta Mater. 154, 14-24 (2018).

50. Li, F. et al. Ultrahigh piezoelectricity in ferroelectric ceramics by design. Nat. Mater. 17, 349-354 (2018).

51. Rossler, S. et al. Two types of magnetic shape-memory effects from twinned microstructure and magneto-structural coupling in $\mathrm{Fe}_{1+\mathrm{y}} \mathrm{Te}$. Proc. Natl Acad. Sci. USA 116, 16697-16702 (2019).

52. Qi, H. et al. Ultrahigh energy-storage density in $\mathrm{NaNbO}_{3}$-based lead-free relaxor antiferroelectric ceramics with nanoscale domains. Adv. Funct. Mater. 29, 1903877 (2019)

53. Wang, B. Growth and Characterization of Lead Zirconate-Titanate $\left(\mathrm{PbZr}_{1-\mathrm{x}} \mathrm{Ti}_{\mathrm{x}} \mathrm{O}_{3}\right.$ )-Based Novel Piezo-/Ferroelectric Single Crystals (Simon Fraser University, 2016).

54. Wei, X. K. et al. Controlled charging of ferroelastic domain walls in oxide ferroelectrics. ACS Appl. Mater. Interfaces 9, 6539-6546 (2017).

55. Feigl, L. et al. Controlled stripes of ultrafine ferroelectric domains. Nat. Commun. 5, 4677 (2014).

56. Wei, X.-K. et al. Flexible polarization rotation at the ferroelectric/metal interface as a seed for domain nucleation. Phys. Rev. B 98, 020102(R) (2018).

57. Parthasarathy, R. Rapid, accurate particle tracking by calculation of radial symmetry centers. Nat. Methods 9, 724-726 (2012).

58. Wieder, H. H. Electrical behavior of barium titanatge single crystals at low temperatures. Phys. Rev. 99, 1161-1165 (1955).

59. Sluka, T., Tagantsev, A. K., Bednyakov, P. \& Setter, N. Free-electron gas at charged domain walls in insulating $\mathrm{BaTiO}_{3}$. Nat. Commun. 4, 1808 (2013).

60. Gao, P. et al. Atomic-scale mechanisms of ferroelastic domain-wall-mediated ferroelectric switching. Nat. Commun. 4, 2791 (2013).

61. Kornev, I. A., Bellaiche, L., Janolin, P. E., Dkhil, B. \& Suard, E. Phase diagram of $\mathrm{Pb}(\mathrm{Zr}, \mathrm{Ti}) \mathrm{O}_{3}$ solid solutions from first principles. Phys. Rev. Lett. 97, 157601 (2006).

62. Sichuga, D. \& Bellaiche, L. Epitaxial $\mathrm{Pb}(\mathrm{Zr}, \mathrm{Ti}) \mathrm{O}_{3}$ ultrathin films under opencircuit electrical boundary conditions. Phys. Rev. Lett. 106, 196102 (2011).

\section{Acknowledgements}

X.-K.W. acknowledges support by the Deutsche Forschungsgemeinschaft (DFG; German Research Foundation) under Germany's Excellence Strategy-Cluster of Excellence Matter and Light for Quantum Computing (ML4Q) EXC 2004/1-390534769. S.P., Y.N. and L.B. thank the DARPA Grant No. HR0011727183-D18AP00010 (TEE Program), Vannevar Bush Faculty Fellowship (VBFF) Grant No. N00014-20-1-2834 from the Department of Defense, and an Arkansas Research Alliance (ARA) Impact Grant. B.-X.W., Y.-J.X. and Z.-G.Y. acknowledge the U.S. Office of Naval Research (ONR Grant No. N00014-16-1 3106 and No. N00014-21-1-2085) and the Natural Sciences \& Engineering Research Council of Canada (NSERC, Grant No. RGPIN-17-06915) for support. Z.L. thanks the Natural Science Foundation of China (Grant No. 51902244), the China Postdoctoral Science Foundation (Grant No. 2018M643632) and the Natural Science Foundation of Shaanxi Province of China (Grant No. 2019JQ-389) for support. The authors thank N Setter for initial discussion and useful advice, and M. Kruth and D. Meertens for their help in FIB sample preparation.

\section{Author contributions}

X.-K.W. conceived the research idea and coordinated the study together with L.B. and Z.-G.Y. X.-K.W. carried out the electron microscopy experiments and data analysis with support from C.L.J., R.E.D.-B. and J.M. S.P., Y.N. and L.B. performed the effective Hamiltonian Monte Carlo simulations. B.-X.W., Y.-J.X., Z.L. and Z.-G.Y. prepared the PZT crystals, examined the crystal structures using X-ray diffraction, and performed dielectric, ferroelectric, and optical property characterizations. X.-K.W. wrote the manuscript and all authors discussed the results and revised the manuscript.

\section{Funding}

Open Access funding enabled and organized by Projekt DEAL.

\section{Competing interests}

The authors declare no competing interests.

\section{Additional information}

Supplementary information The online version contains supplementary material available at https://doi.org/10.1038/s41467-021-25543-1.

Correspondence and requests for materials should be addressed to X.-K.W., L.B. or Z.-G.Y.

Peer review information Nature Communications thanks Gang Liu, Haijun Wu and the other, anonymous, reviewer(s) for their contribution to the peer review of this work

Reprints and permission information is available at http://www.nature.com/reprints

Publisher's note Springer Nature remains neutral with regard to jurisdictional claims in published maps and institutional affiliations.

Open Access This article is licensed under a Creative Commons Attribution 4.0 International License, which permits use, sharing, adaptation, distribution and reproduction in any medium or format, as long as you give appropriate credit to the original author(s) and the source, provide a link to the Creative Commons license, and indicate if changes were made. The images or other third party material in this article are included in the article's Creative Commons license, unless indicated otherwise in a credit line to the material. If material is not included in the article's Creative Commons license and your intended use is not permitted by statutory regulation or exceeds the permitted use, you will need to obtain permission directly from the copyright holder. To view a copy of this license, visit http://creativecommons.org/ licenses/by/4.0/.

(C) The Author(s) 2021 